\title{
Inter-rater agreement on proximal humerus fracture treatment in Korea
}

\author{
Chris Hyunchul Jo \\ Department of Orthopedic Surgery, SMG-SNU Boramae Medical Center, Seoul National University College of Medicine, Seoul, Korea
}

Proximal humerus fracture is the third most common fracture, after distal radius fracture and proximal femur fracture; it affects a considerable number of adult and elderly patients due to trauma or falls [1]. The prevalence of proximal humerus fracture in hospital emergency care is substantial and corresponds to approximately $80 \%$ of humerus fractures and $5 \%$ of total fractures [2]. This prevalence continues to rise with extended life span and increased outdoor activity.

A standardized classification system is needed for several purposes including communication between medical professionals, standardization of research, and, most importantly, for use in prognostication and to guide management and intervention. However, inter-rater agreement for classification and treatment selection of proximal humerus fractures is quite low, and decision making for the treatment of these fractures is challenging even for experienced surgeons [3,4]. To overcome these difficulties, several approaches, such as use of multiple Neer classifications, AO/OTA (Orthopaedic Trauma Association) [5], three-dimensional computed tomography (3D CT) [6], 3D handheld modeling [4,7], and artificial intelligence [8] have been reported. Thus, research on current approaches to treatment of proximal humerus fracture would be helpful to surgeons.

A study by Kim et al. [9] in Clinics in Shoulder and Elbow investigated inter-rater and intra-rater agreement with respect to selection of treatment method for proximal humerus fractures among fellowship-trained shoulder surgeons with at least 5 years of clinical experience. The participating surgeons assessed 40 proximal humerus fractures with two X-rays and one CT image and answered three questions in the first stage of classification and selection between conservative and surgical management, as well as an additional three questions in the second stage where either conservative or surgical options were specified. The results showed that inter-rater agreement for fracture classification was fair to moderate (Fleiss' kappa of 0.395 for the first view and 0.417 for the second view), moderate for selection between conservative and surgical treatment (kappa of 0.528 and 0.417 ), and substantial for specification of surgical options (kappa of 0.740 and 0.727 ). These results are in line with previous studies with respect to inter-rater agreement for the classification and selection of treatment method for proximal humerus fractures [5-7]. Meanwhile, the low inter-rater agreement with kappa of 0.395 0.417 was notable, as 3D CT was also used in this study. This was likely due to the provision of a single 3D CT image rather than a full sequence, suggesting that multiple 3D CT images may be helpful for improving classification agreement.

Taken together, this evidence indicates that moderate to substantial agreement was achieved with Neer classification using $\mathrm{X}$-rays and 3D CT, but also suggests that further tools and studies

Received: February 2, 2022 Accepted: February 16, 2022

Correspondence to: Chris Hyunchul Jo

Department of Orthopedic Surgery, SMG-SNU Boramae Medical Center, Seoul National University College of Medicine, 20 Boramae-ro 5-gil, Dongjakgu, Seoul 07061, Korea

Tel: +82-2-840-2453, Fax: +82-2-870-3864, E-mail: chrisjo@snu.ac.kr, ORCID: https://orcid.org/0000-0002-6161-5442

Financial support: None.

Conflict of interest: None.

Copyright@ 2022 Korean Shoulder and Elbow Society.

This is an Open Access article distributed under the terms of the Creative Commons Attribution Non-Commercial License (http://creativecommons.org/licenses/by-nc/4.0/) which permits unrestricted non-commercial use, distribution, and reproduction in any medium, provided the original work is properly cited. 
are needed for further improvement of agreement.

\section{ORCID}

\section{Chris Hyunchul Jo}

https://orcid.org/0000-0002-6161-5442

\section{REFERENCES}

1. Robinson BC, Athwal GS, Sanchez-Sotelo J, Rispoli DM. Classification and imaging of proximal humerus fractures. Orthop Clin North Am 2008;39:393-403.

2. de Oliveira AP, Mestieri MC, Pontin JC. Epidemiological profile of patients with proximal humerus fracture treated at Hospital São Paulo, Brazil. Acta Ortop Bras 2015;23:271-4.

3. Stirma GA, Secundino AR, Gonzalez GF, Sola WC, de Souza GA, Dau L. Inter/intra-observer evaluation between radiographs and tomographies for Proximal humerus fracture. Acta Ortop Bras 2020;28:36-9.

4. Cocco LF, Aihara AY, Lopes FP, et al. Three-dimensional printing models increase inter-rater agreement for classification and treatment of proximal humerus fractures. Patient Saf Surg
2022;16:5.

5. Wennergren D, Stjernström S, Möller M, Sundfeldt M, Ekholm C. Validity of humerus fracture classification in the Swedish fracture register. BMC Musculoskelet Disord 2017;18:251.

6. Cocco LF, Yazzigi JA Jr, Kawakami EF, Alvachian HJ, Dos Reis FB, Luzo MV. Inter-observer reliability of alternative diagnostic methods for proximal humerus fractures: a comparison between attending surgeons and orthopedic residents in training. Patient Saf Surg 2019;13:12.

7. Spek RW, Schoolmeesters BJ, Oosterhoff JH, et al. 3D-printed handheld models do not improve recognition of specific characteristics and patterns of three-part and four-part proximal humerus fractures. Clin Orthop Relat Res 2022;480:150-9.

8. Chung SW, Han SS, Lee JW, et al. Automated detection and classification of the proximal humerus fracture by using deep learning algorithm. Acta Orthop 2018;89:468-73.

9. Kim H, Song SJ, Jeon IH, Koh KH. Inter-rater agreement among shoulder surgeons on treatment options for proximal humeral fractures among shoulder surgeons. Clin Shoulder Elbow 2022;25:49-56. 